\title{
The Dust Properties of Eight Debris Disk Candidates as Determined by Submillimeter Photometry
}

\author{
Jonathan P. Williams and Sean M. Andrews \\ Institute for Astronomy, University of Hawaii, 2680 Woodlawn Drive, Honolulu, HI 96822 \\ jpw@ifa.hawaii.edu, andrews@ifa.hawaii.edu
}

\begin{abstract}
The nature of far-infrared dust emission toward main sequence stars, whether interstellar or circumstellar, can be deduced from submillimeter photometry. We present JCMT/SCUBA flux measurements at $850 \mu \mathrm{m}$ toward 8 stars with large photospheric excesses at $60-100 \mu \mathrm{m}$. 5 sources were detected at $3 \sigma$ or greater significance and one was marginally detected at $2.5 \sigma$. The inferred dust masses and temperatures range from 0.033 to $0.24 M_{\oplus}$ and $43-65 \mathrm{~K}$ respectively. The frequency behavior of the opacity, $\tau_{\nu} \propto \nu^{\beta}$, is relatively shallow, $\beta<1$. These dust properties are characteristic of circumstellar material, most likely the debris from planetesimal collisions. The 2 non-detections have lower temperatures, $35-$ $38 \mathrm{~K}$, and steeper opacity indices, $\beta>1.5$, that are more typical of interstellar cirrus. The confirmed disks all have inferred diameters $\gtrsim 2^{\prime \prime}$ most lie near the upper envelope of the debris disk mass distribution, and 4 are bright enough to be feasible for high resolution imaging.
\end{abstract}

Subject headings: circumstellar matter — planetary systems: protoplanetary disks — planetary systems: formation — submillimeter

\section{Introduction}

Dust around main sequence stars with ages $\gtrsim 10$ Myr must have been formed in situ due to the short timescales for collisional destruction and radiative dispersal (Artymowicz 1988). The micron and sub-micron sized particles that are detected in infrared images of scattered light (e.g., Liu 2004) and emission (e.g., Su et al. 2005) are created from the collisions of larger particles, possibly from the late stages of planet formation. The study of such debris disks, therefore, constrain planet formation models (Kenyon \& Bromley 2004) and new detections can signpost good targets for imaging of extrasolar planets (Zuckerman \& Song 2004). 
The faint emission from the cold dust is generally only apparent above the stellar photosphere at wavelengths longer than about $20 \mu \mathrm{m}$. The IRAS all sky survey has been the primary resource for debris disk searches, subsequently augmented with more targeted surveys by ISO (see de Muizon 2005) and most recently by Spitzer (Meyer et al. 2004; Rieke et al. 2005). These instruments are able to define the spectral energy distribution (SED) of the dust out to near its peak at about $100 \mu \mathrm{m}$, but photometry at longer wavelengths where the emission is optically thin is an essential complement by providing a measure of the dust mass. Moreover, by constraining the Rayleigh-Jeans side of the SED, the disk temperature and luminosity and the the power law index of the dust opacity can be determined. These are significantly different in debris disks and the interstellar medium and thus submillimeter observations can confirm or reject debris disk candidates that are based solely on unresolved far-infrared excesses.

Observations of debris disks at long wavelengths are difficult due to the intrinsic weakness of the emission and the poor transparency of the atmosphere. The Submillimeter Common-User Bolometer Array (SCUBA) on the James Clerk Maxwell Telescope (JCMT) has been the workhorse instrument to date (e.g., Holland et al. 1998) but has still had limited success: less than 20 systems have been detected at submillimeter wavelengths. Consequently, the evolution of mass and inner radius, derived from the dust temperature, is not well understood (Najita \& Williams 2005).

Far-infrared surveys provide a good starting point to define a target list for submillimeter observations, although the coldest disks may be missed (Wyatt, Dent, \& Greaves 2003; Najita \& Williams 2005). A recent list of 58 nearby $(<100$ pc) debris disk candidates was published by Zuckerman \& Song (2004) based on an extensive search of the IRAS Faint Source Catalog by Silverstone (2000). Most of their candidates have not been observed beyond $100 \mu \mathrm{m}$ but many have large excesses and their SEDs extrapolate to detectable fluxes in the submillimeter regime.

A program to obtain $850 \mu \mathrm{m}$ photometry of stars in the Zuckerman \& Song catalog began in Spring 2004 with the intent to survey as many sources with large predicted fluxes as possible. The unfortunate retirement of SCUBA in the summer of 2005 ended the project after only 8 sources had been observed. Nevertheless, the truncated survey yielded a very high $(75 \%)$ detection rate and significant constraints on the nature of the dust toward the 2 other sources. This short paper describes the observations in $\S 2$, the spectral energy distributions of the 8 candidate debris disks in $\S 3$, discusses the results and implications in $\S 4$, and concludes in $\S 5$. 


\section{Observations}

Photometry observations were conducted using the SCUBA bolometer at the JCMT on Mauna Kea, Hawaii between March 2004 and July 2005. For most of the data presented here, the precipitable water vapor level was between 1 and $2 \mathrm{~mm}$ corresponding to zenith optical depths at $850 \mu \mathrm{m}$ between about 0.2 to 0.3 . The optical depth at $450 \mu \mathrm{m}$ was generally greater than 1 so high sensitivity measurements were not possible with the short wavelength bolometers.

Each source, whose position was taken from the 2MASS Point Source Catalog, was observed for between 50 and 70 minutes with the exception of HD 206893 which was observed for 140 minutes. The pointing was checked via observations of bright quasars near each source after each major slew and the focus was adjusted every three hours on average, more frequently at times near sunrise and sunset. Calibration was performed by observations of Uranus, Mars and standard sources, CRL 618, CRL 2688, IRC+10216. Based on the agreement of the measured fluxes with those predicted for these calibrators, we estimate the photometry is accurate to within $10 \%$ at $850 \mu \mathrm{m}$.

$\mathrm{CO}(3-2)$ emission was detected toward HD 218396 using the B3 dual polarization receiver on the JCMT during observations in June 2005. The emission was relatively strong and a small map was made in the vicinity of the source to measure its extent. The map was made by scanning across the field alternately in right ascension and declination with a fixed off position $3^{\prime}$ east of the map center. The system temperatures decreased from $620 \mathrm{~K}$ to $430 \mathrm{~K}$ as the source rose from $37^{\circ}$ to $64^{\circ}$. First order baselines were removed and the data gridded and coadded to produce a fully sampled map with size $70^{\prime \prime} \times 70^{\prime \prime}$ and $\mathrm{rms} 0.2 \mathrm{~K}$ per $0.15 \mathrm{~km} \mathrm{~s}^{-1}$ channel. Due to the time taken to make this map, none of the other sources were observed with the heterodyne receivers.

\section{Results}

\subsection{Spectral Energy Distributions}

The 8 sources that were observed with SCUBA are listed in Table 1 . The age estimates are from a recent re-evaluation by Moór et al. (2006) whose target list of 60 debris disk candidates with high fractional luminosity overlap with 6 of the sources here. The ages for the two sources not in Moór et al., HD 14055 and HD 56099, are taken from Zuckerman \& Song (2004). Distances are derived from Hipparcos parallaxes. The SCUBA flux measurements and $1 \sigma$ statistical uncertainty are listed in the last column of the table. A $10 \%$ calibration uncertainty applies to both the flux and error. 
Five of the eight sources, HD 14055, HD 15115, HD 21997, HD 127821, and HD 219396 were detected at $850 \mu \mathrm{m}$ at $3 \sigma$ significance or higher. An additional source, HD 206893, was detected with a signal-to-noise ratio of 2.5. It was observed on two consecutive days with consistent $(\sim 2 \sigma)$ results and is considered a marginal detection. The SEDs of these six sources are shown in Figure 1. The Hipparcos and 2MASS catalogs were used to obtain the photometry at BV and JHK bands respectively and Kurucz models appropriate for each stellar type are shown normalized to the near-infrared data. Fluxes at mid- and far-infrared wavelengths are from the IRAS Faint Source Catalog, color corrected for the temperature of the star at 12 and $25 \mu \mathrm{m}$ and for the temperature of the dust at 60 and $100 \mu \mathrm{m}$ where appropriate. Since the corrections change the SED fit slightly they are determined iteratively along with the dust temperature. Additional data points from ISO and Spitzer observations at $10-90 \mu \mathrm{m}$ from Chen et al. (2006) and Moór et al. (2006) were added where available.

We model the excess dust emission above the stellar photosphere with a single temperature greybody, $F_{\nu}=B_{\nu}\left(T_{\text {dust }}\right)\left(1-e^{-\tau_{\nu}}\right) \Delta \Omega$. Here $B_{\nu}$ is the Planck function, $\tau_{\nu}=\left(\nu / \nu_{0}\right)^{\beta}=$ $\left(\lambda / \lambda_{0}\right)^{-\beta}$ is the optical depth with power law index $\beta$, and $\Delta \Omega$ is the solid angle. Because the dust is only detected at $3-6$ wavelengths in each source, we apply an additional constraint, $\lambda_{0} \leq 100 \mu \mathrm{m}$. This is based on similar fits to better sampled SEDs of other debris disks (Dent et al. 2000) and is also justified a posteriori from the fitted disk parameters below.

The dust excesses were then fit to determine $\beta, T_{\text {dust }}$, and $\Delta \Omega$ for a range of values of $\lambda_{0}$. The results are shown for a representative source, HD 15115, in Figure 2. Fits to the SEDs of the other sources show a similar behavior: $\beta$ and $T_{\text {dust }}$ decrease slightly as $\lambda_{0}$ decreases but $\Delta \Omega$ increases rapidly. This is due to the fact that the dust is cold, $\sim 50 \mathrm{~K}$, so the midand far-infrared data points all lie on the Wien side of the greybody where the frequency dependence is dominated by the exponential term. The optical depth dependence produces only a slight change to the shape of the fit and, if less than one, mainly affects the flux scale.

The different fits for the different values of $\lambda_{0}$ are almost indistinguishable in terms of the least squares difference and the plotted SED, shown in Figure 1. Consequently these data are unable to give an accurate measure of $\lambda_{0}$ and therefore also $\Delta \Omega$. Because $\beta$ and $T_{\text {dust }}$ are only weakly dependent on $\lambda_{0}$, however, they are well determined. The one SCUBA data point is critical in this regard because it lies on the Rayleigh-Jeans side of the distribution where the emission is both optically thin (so $\lambda_{0}$ is only a scaling factor), and the frequency dependence is a power law and therefore strongly dependent on $\beta$.

The remaining two sources in the observed sample, HD 56099 and HD 78702, were not detected. Both stars have strong infrared excesses but the low limit on the $850 \mu \mathrm{m}$ flux from the SCUBA observations implies a significantly steeper submillimeter spectral index, or equivalently a higher value for $\beta$, than determined for the detected sources. The best 
three-parameter fit to the observed IRAS and SCUBA fluxes requires $\beta \geq 2$ for all values of $\lambda_{0}$. Mie theory, laboratory measurements, and ISM observations suggest an upper limit of $\beta=2$ (Pollack et al. 1994; Draine 2006). The lowest value of $\beta$ consistent with the data is obtained by fitting the SED with an $850 \mu \mathrm{m}$ flux equal to the $3 \sigma$ SCUBA upper limit. The best fit SED is shown for each source in Figure 3, and the allowable fits consistent with the upper limits are overlaid in greyscale.

Table 2 lists the best fit parameters, $T_{\text {dust }}$ and $\beta$, and their full range, including errors, for $\lambda_{0}=3-100 \mu \mathrm{m}$. The derived dust mass and fractional luminosity are listed in columns 3 and 4 respectively. The dust mass is calculated from the $850 \mu \mathrm{m}$ flux in the standard way and assumes a dust mass absorption coefficient $\kappa_{850}=1.7 \mathrm{~cm}^{2} \mathrm{~g}^{-1}$ for ease of comparison with other studies. There is considerable uncertainty over this number and the masses of the submillimeter emitting particles may be underestimated by a factor of $3-5$ (see discussion in Najita \& Williams 2005). This uncertainty dominates that due to the linear dependence of mass on $T_{\text {dust }}$. The ratio of dust to stellar luminosity is a measure of the fraction of the starlight absorbed by the dust and is a good indicator of the likelihood of detecting scattered light (see $\S 4$ ). This value is independent of $\lambda_{0}$ as the plotted best fit SEDs are almost indistinguishable. The fact that a single temperature characterizes the emission very well for each SCUBA detection shows that that the mass surface density jumps at a specific distance from the star $^{1}$. A minimum radius of this inner hole, $R_{\text {dust }}=7.8 \times 10^{4} \mathrm{AU}\left(L_{\text {star }} / L_{\odot}\right)^{0.5} T_{\text {dust }}^{-2}$, is obtained by bounding the dust luminosity by a blackbody and is listed in column six of Table 2 .

The low masses and large radii that we have determined allow us to justify, a posteriori, our initial assumption in the SED fits. Even if the dust were concentrated in a narrow annulus of width $\Delta R_{\text {dust }}=1 \mathrm{AU}$ at a radius $R_{\text {dust }}$ from the star, the inferred surface densities for each source are very small, $\Sigma_{\text {dust }}=M_{\text {dust }} / 2 \pi R_{\text {dust }} \Delta R_{\text {dust }} \simeq 10^{-3}-10^{-2} \mathrm{~g} \mathrm{~cm}^{-2}$. Since $\tau_{\lambda} \propto \lambda^{-\beta}$, the wavelength where the disks become optically thin is $\lambda_{0}=850 \mu \mathrm{m}\left(\kappa_{850} \Sigma_{\text {dust }}\right)^{1 / \beta} \simeq 1-$ $10 \mu \mathrm{m}$. If the dust were more spread out, or the inner hole larger than the minimum estimate in Table 2 , the inferred value of $\lambda_{0}$ would be even smaller.

The five detections and one marginal detection have dust temperatures ranging from $43-65 \mathrm{~K}$ and dust opacity index $\beta=0.6-1$. These are typical values for debris around main sequence stars (Dent et al. 2000; Najita \& Williams 2005) and different from the diffuse interstellar medium where $T_{\text {dust }} \approx 20 \mathrm{~K}$ and $\beta \approx 2$ (Boulanger et al. 1996). Thus these SCUBA observations help to confirm the disk nature of the far-infrared emission around

\footnotetext{
${ }^{1}$ There may still be a significant amount of dust, as measured by area, in very small dust grains closer to the star. Scattered light images need not, therefore, show a central hole (Wahhaj et al. 2005).
} 
these stars.

On the other hand, the possible fits to the two non-detections have lower temperatures, $T_{\text {dust }}=36-38 \mathrm{~K}$, and steeper dust opacity indices $\beta>1.5$ than the other sources in the sample. These values suggest that the IRAS emission is warm interstellar cirrus (Low et al. 1984) and we conclude that these two sources are probably not debris disks.

\subsection{Molecular gas along the line of sight to HD 218396}

A CO 3-2 spectrum was taken toward HD 218396 and surprisingly strong emission was detected. CO disk emission rapidly decreases as the infrared excess decreases (Dent, Greaves, \& Coulson 2005). No star with such a small excess has been detected in CO before and the measured integrated intensity, $I_{\mathrm{CO}}=1.6 \mathrm{~K} \mathrm{~km} \mathrm{~s}^{-1}$, is more typical of protostellar, rather than debris, disks.

In fact, it seems unlikely that the $\mathrm{CO}$ comes from a disk around the star. The radial velocity of the gas, $v_{\mathrm{CO}}=-5.0 \mathrm{~km} \mathrm{~s}^{-1}$ and star, $v_{*}=-12.6 \mathrm{~km} \mathrm{~s}^{-1}$ (Moór et al. 2006), are significantly different and there is no indication that the star is a spectroscopic binary (Royer et al. 2002). No significant emission to a $3 \sigma$ limit of $0.54 \mathrm{~K}$ per $0.15 \mathrm{~km} \mathrm{~s}^{-1}$ channel is detected at the stellar radial velocity. We also mapped the emission and found it to be extended (Figure 4). The CO line probably arises from an unrelated background high latitude $\left(b=-35.6^{\circ}\right)$ cloud along the line of sight, about $1^{\circ}$ north and perhaps an extension of cloud 54 in the catalog of Magnani, Blitz, \& Mundy (1985). The filling factor of high latitude molecular gas is small, $\approx 3 \%$, in the southern hemisphere (Magnani et al. 2000), but not negligible.

The mid-infrared emission is compact in the Spitzer IRS SL slit, at $6^{\prime \prime}$ resolution, but it may possess some extended emission, containing approximately half the total flux, in the wings of the PSF in the LL2 slit (Chen, personal communication). At the much lower IRAS resolution, $\simeq 1^{\prime}$, there is no evidence for extended emission. The SCUBA detection of HD 218396 has the highest signal-to-noise ratio, $\simeq 6$, of our sample. There is no emission in the neighboring bolometers, however, showing that the $850 \mu \mathrm{m}$ flux is concentrated within about $20^{\prime \prime}$.

Any extended background emission would imply that the measured fluxes overestimate the disk emission. A uniform scaling would not change the inferred values of $T_{\text {dust }}$ and $\beta$. The shallow submillimeter slope, in particular, is strong evidence that the dust emission is disk-like in nature. Even if the SCUBA flux were a factor of 2 lower and the infrared data unchanged, the best fit, $\beta=1$, is significantly less than ISM dust. 
Based on the above, we conclude that HD 218396 is surrounded by a debris disk that is detected in the continuum from $30-850 \mu \mathrm{m}$, but not in the CO 3-2 line.

\section{Discussion}

The 8 sources here were selected based on the extrapolation of far-infrared SEDs to submillimeter wavelengths. 6 of the 8 were detected, although one only at $2.5 \sigma$ significance. This is a far higher success rate than other submillimeter surveys based solely on young stellar ages, either via association with massive stars (Wyatt et al. 2003) or moving group membership (Liu et al. 2004) and stellar activity (Najita \& Williams 2005). The stellar age of the 6 detected sources range from $\sim 10 \mathrm{Myr}$ to $\gtrsim 200 \mathrm{Myr}$ and possibly as large as $3 \mathrm{Gyr}$. Rieke et al. (2005) suggest that age is not the only factor in either disk occurrence or dust mass and that there may be a significant stochastic influence due to rare collisions of large objects (see also Song et al. 2005).

Carpenter et al. (2005) showed that the mass of dust particles traced by millimeter measurements is smaller in debris disks around main sequence stars than protostellar disks. Liu et al. (2004) and Najita \& Williams (2005) define an upper envelope to the debris disk mass distribution that decreases inversely with age. The new detections here and the revised age estimates in Moór et al. (2006) do not greatly alter these conclusions (Figure 5). 4 of the 6 new detections here lie near the upper boundary of the mass distribution and two, HD 127821 and HD 206893, add to the statistics in the poorly characterized region where stellar ages are greater than $300 \mathrm{Myr}$.

We have also reexamined the dependence of dust temperature and minimum inferred radius on the stellar luminosity and age with the addition of the 6 new sources here. As in Najita \& Williams (2005), no obvious correlations were found.

The double peak in the SEDs shows that the dust is physically separated from the stars but the far-infrared and submillimeter observations discussed here are unable to resolve the disks. Higher resolution studies are important for measuring disk sizes and characterizing asymmetries that may signpost planetary companions. Minimum radii based on the fitted dust temperatures are given in Table 2 and range from $1-2^{\prime \prime}$ in angular size. These are well within the range of optical and infrared observations and the issue of detectability is one of contrast. Two systems, HD 15115 and HD 21997, have reasonably high fractional luminosities, $L_{\text {dust }} / L_{\text {star }}=6-7 \times 10^{-4}$, that are comparable to AU Mic and therefore might be detectable in scattered light either via ground based coronagraphy (Kalas, Liu, \& Matthews 2004; Liu 2004) or direct imaging with the Hubble Space Telescope (e.g., Krist et al. 2005).

Interferometric imaging at (sub)millimeter wavelengths is an important alternative means 
to investigate disk structure (e.g., Wilner et al. 2002). The millimeter sized particles that are revealed in such observations are less affected by radiative forces than the micron sized particles seen in scattered light. Consequently, resolved millimeter wavelength images are a more reliable measure of the gravitational potential of a system and of any dynamical resonances that might be induced by a planet (Kuchner \& Holman 2003; Wyatt 2003). The two systems here, HD 127821 and HD 218396, with $850 \mu \mathrm{m}$ fluxes greater than $10 \mathrm{mJy}$, and diameters $\gtrsim 3-4^{\prime \prime}$, are just accessible to the current generation of interferometers for high resolution mapping.

\section{Conclusions}

We have carried out $850 \mu \mathrm{m}$ photometry of 8 nearby stars to search for thermal emission from cold circumstellar dust. With one exception, the rms noise levels of these SCUBA observations lie between $\sigma=1.5-2.3 \mathrm{mJy}$. 5 sources were detected at $3 \sigma$ significance or higher and an additional one at $2.5 \sigma$. The high detection rate is attributable to their selection based on high IRAS 60 and $100 \mu \mathrm{m}$ excesses.

These 6 sources are a significant addition to the small number of debris disks detected at submillimeter wavelengths. The inferred dust masses range from $0.033-0.23 M_{\oplus}$. Several are some of the most massive disks known for their age and show that even the upper end of the disk mass distribution may not have been fully explored. Dust temperatures range from $43-65 \mathrm{~K}$, and are similar to other debris disks. There is no correlation of either temperature of equivalent black-body radius with stellar luminosity or age.

Far-infrared and submillimeter observations are generally unable to resolve the emission from the cold dust. Flux measurements, or even sensitive upper limits, on the Rayleigh-Jeans side of the SED regime constrain the dust opacity index and are important for distinguishing between a disk or interstellar (most likely cirrus) origin. The former applies for the 6 detections which have $\beta<1$ and the latter for the 2 non-detections where the combination of high far-infrared and low submillimeter fluxes constrain $\beta>1.5$.

The photospheric excesses are well fit by single temperature greybodys implying that the bulk of the dust, as measured by mass, lies far from the star, ranging from 42 to 84 AU for each source or about $1-2^{\prime \prime}$ in angular size. Two of the confirmed disks have high fractional luminosities and may be detectable in scattered light. An additional 2 are strong enough to image with the current generation of (sub)millimeter interferometers. Such high resolution observations may show clumping in the disk due to planetary induced resonances.

There are undoubtedly more sources in the Zuckerman \& Song catalog, and perhaps

others in the plethora of new Spitzer results (e.g., Bryden et al. 2006), that would be 
detectable if SCUBA were available. Its successor, SCUBA-2, will be about a factor of 3 times more sensitive for photometry and far quicker for mapping (Holland et al. 2003). It will distinguish between cirumstellar and interstellar emission and provide mass measurements and dust opacities for perhaps as many as an order of magnitude more debris disks.

This work is supported by the NSF through grant AST-0324328. We thank Christine Chen and Mike Jura for helpful discussions concerning Spitzer IRS photometry, and Michael Liu and Joan Najita for comments on the manuscript. This research has made use of the SIMBAD database and the Two Micron All Sky Survey, which is a joint project of the University of Massachusetts and IPAC/Caltech, funded by NASA and NSF.

\section{REFERENCES}

Artymowicz, P. 1988, ApJ, 335, L79

Bryden, G., et al. 2006, ApJ, 636, 1098

Boulanger, F., Abergel, A., Bernard, J.-P., Burton, W. B., Desert, F.-X., Hartmann, D., Lagache, G., \& Puget, J.-L. 1996, A\&A, 312, 256

Carpenter, J. M., Wolf, S., Schreyer, K., Launhardt, R., \& Henning, T. 2005, AJ, 129, 1049

Chen, C. H., et al. 2006, ApJS, in press (astro-ph/0605277)

de Muizon, M. J. 2005, Space Science Reviews, 119, 201

Dent, W. R. F., Walker, H. J., Holland, W. S., \& Greaves, J. S. 2000, MNRAS, 314, 702

Dent, W. R. F., Greaves, J. S., \& Coulson, I. M. 2005, MNRAS, 359, 663

Draine, B. T. 2006, ApJ, 636, 1114

Greaves, J. S., Wyatt, M. C., Holland, W. S., \& Dent, W. R. F. 2004, MNRAS, 351, L54

Holland, W. S., et al. 1998, Nature, 392, 788

Holland, W. S., Duncan, W., Kelly, B. D., Irwin, K. D., Walton, A. J., Ade, P. A. R., \& Robson, E. I. 2003, Proc. SPIE, 4855, 1

Kalas, P., Liu, M. C., \& Matthews, B. C. 2004, Science, 303, 1990

Krist, J. E., et al. 2005, AJ, 129, 1008

Kenyon, S. J. \& Bromley, B. C. 2004, AJ, 127, 513

Kuchner, M. J., \& Holman, M. J. 2003, ApJ, 588, 1110

Liu, M. C. 2004, Science, 305, 1442 
Liu, M. C., Matthews, B. C., Williams, J. P., \& Kalas, P. G. 2004, ApJ, 608, 526

Low, F. J., et al. 1984, ApJ, 278, L19

Magnani, L., Blitz, L., \& Mundy, L. 1985, ApJ, 295, 402

Magnani, L., Hartmann, D., Holcomb, S. L., Smith, L. E., \& Thaddeus, P. 2000, ApJ, 535, 167

Meyer, M. R., et al. 2004, ApJS, 154, 422

Moór, A., Ábrahám, P., Derekas, A., Kiss, C., Kiss, L. L., Apai, D., Grady, C., \& Henning, T. 2006, ApJ, 644, 525

Najita, J., \& Williams, J. P. 2005, ApJ, 635, 625

Pollack, J. B., Hollenbach, D., Beckwith, S., Simonelli, D. P., Roush, T., \& Fong, W. 1994, ApJ, 421, 615

Rieke, G. H., et al. 2005, ApJ, 620, 1010

Royer, F., Grenier, S., Baylac, M.-O., Gómez, A. E., \& Zorec, J. 2002, A\&A, 393, 897

Sheret, I., Dent, W. R. F., Wyatt, M. C. 2004, MNRAS, 348, 1282

Silverstone, M. D. 2000, Ph.D. Thesis, UCLA

Song, I., Zuckerman, B., Weinberger, A. J., \& Becklin, E. E. 2005, Nature, 436, 363

Su, K. Y. L., et al. 2005, ApJ, 628, 487

Wahhaj, Z., Koerner, D. W., Backman, D. E., Werner, M. W., Serabyn, E., Ressler, M. E., \& Lis, D. C. 2005, ApJ, 618, 385

Williams, J. P., Najita, J., Liu, M. C., Bottinelli, S., Carpenter, J. M., Hillenbrand, L. A., Meyer, M. R., \& Soderblom, D. R. 2004, ApJ, 604, 414

Wilner, D. J., Holman, M. J., Kuchner, M. J., \& Ho, P. T. P. 2002, ApJ, 569, L115

Wyatt, M. C., Dent, W. R. F., \& Greaves, J. S. 2003, MNRAS, 342, 876

Wyatt, M. C., Greaves, J. S., Dent, W. R. F., \& Coulson I. M. 2005, ApJ, 620, 492

Wyatt, M. C. 2003, ApJ, 598, 1321

Zuckerman, B., \& Song, I. 2004, ApJ, 603, 738 
TABLE 1

Sourcelist and submillimeter photometry

\begin{tabular}{lccccc}
\hline Source & HIP & SpT & $\begin{array}{c}\text { Age } \\
(\mathrm{Myr})\end{array}$ & $\begin{array}{c}d \\
(\mathrm{pc})\end{array}$ & $\begin{array}{c}F_{850} \\
(\mathrm{mJy})\end{array}$ \\
\hline \hline HD 14055 & 10670 & A1V & $100 ?$ & 36.1 & $5.5 \pm 1.8$ \\
HD 15115 & 11360 & F2 & $12_{-4}^{+8}$ & 44.8 & $4.9 \pm 1.6$ \\
HD 21997 & 16449 & A3IV /V & $20_{-10}^{+10}$ & 73.8 & $8.3 \pm 2.3$ \\
HD 56099 & 35457 & F8 & $>500 ?$ & 86.8 & $1.4 \pm 1.9$ \\
HD 78702 & 44923 & A0/A1V & $220_{-140}^{+100}$ & 79.9 & $0.3 \pm 2.3$ \\
HD 127821 & 70952 & F4IV & {$[200,3400]$} & 31.7 & $13.2 \pm 3.7$ \\
HD 206893 & 107412 & F5V & $<2800$ & 38.9 & $3.8 \pm 1.5$ \\
HD 218396 & 114189 & A5V & {$[20-150]$} & 39.9 & $10.3 \pm 1.8$ \\
\hline
\end{tabular}

TABLE 2

Dust properties

\begin{tabular}{lllrrrl}
\hline Source & $\begin{array}{l}T_{\text {dust }} \\
(\mathrm{K})\end{array}$ & $\beta$ & $\begin{array}{r}M_{\text {dust }} \\
\left(M_{\oplus}\right)\end{array}$ & $\begin{array}{r}L_{\text {dust }} / L_{\text {star }} \\
\left(10^{-4}\right)\end{array}$ & $\begin{array}{l}R_{\text {dust }} \\
(\mathrm{AU})\end{array}$ & Notes \\
\hline \hline HD 14055 & $65_{-10}^{+4}$ & $0.98_{-0.30}^{+0.17}$ & 0.033 & 1.0 & 84 & \\
HD 15115 & $62_{-5}^{+3}$ & $0.73_{-0.27}^{+0.16}$ & 0.047 & 5.8 & 34 & \\
HD 21997 & $56_{-6}^{+1}$ & $0.86_{-0.27}^{+0.14}$ & 0.24 & 7.1 & 75 & \\
HD 56099 & $38_{-5}$ & $2.00_{-0.51}$ & $<0.091$ & $\sim 11$ & & interstellar cirrus? \\
HD 78702 & $36_{-7}$ & $2.00_{-0.19}$ & $<0.018$ & $\sim 2.6$ & & interstellar cirrus? \\
HD 127821 & $43_{-9}^{+10}$ & $0.64_{-0.34}^{+0.44}$ & 0.096 & 3.0 & 67 & \\
HD 206893 & $53_{-10}^{+5}$ & $0.82_{-0.36}^{+0.25}$ & 0.033 & 2.8 & 42 & marginal detection \\
HD 218396 & $50_{-3}^{+1}$ & $0.71_{-0.21}^{+0.10}$ & 0.10 & 2.8 & 66 & CO detection \\
\hline
\end{tabular}



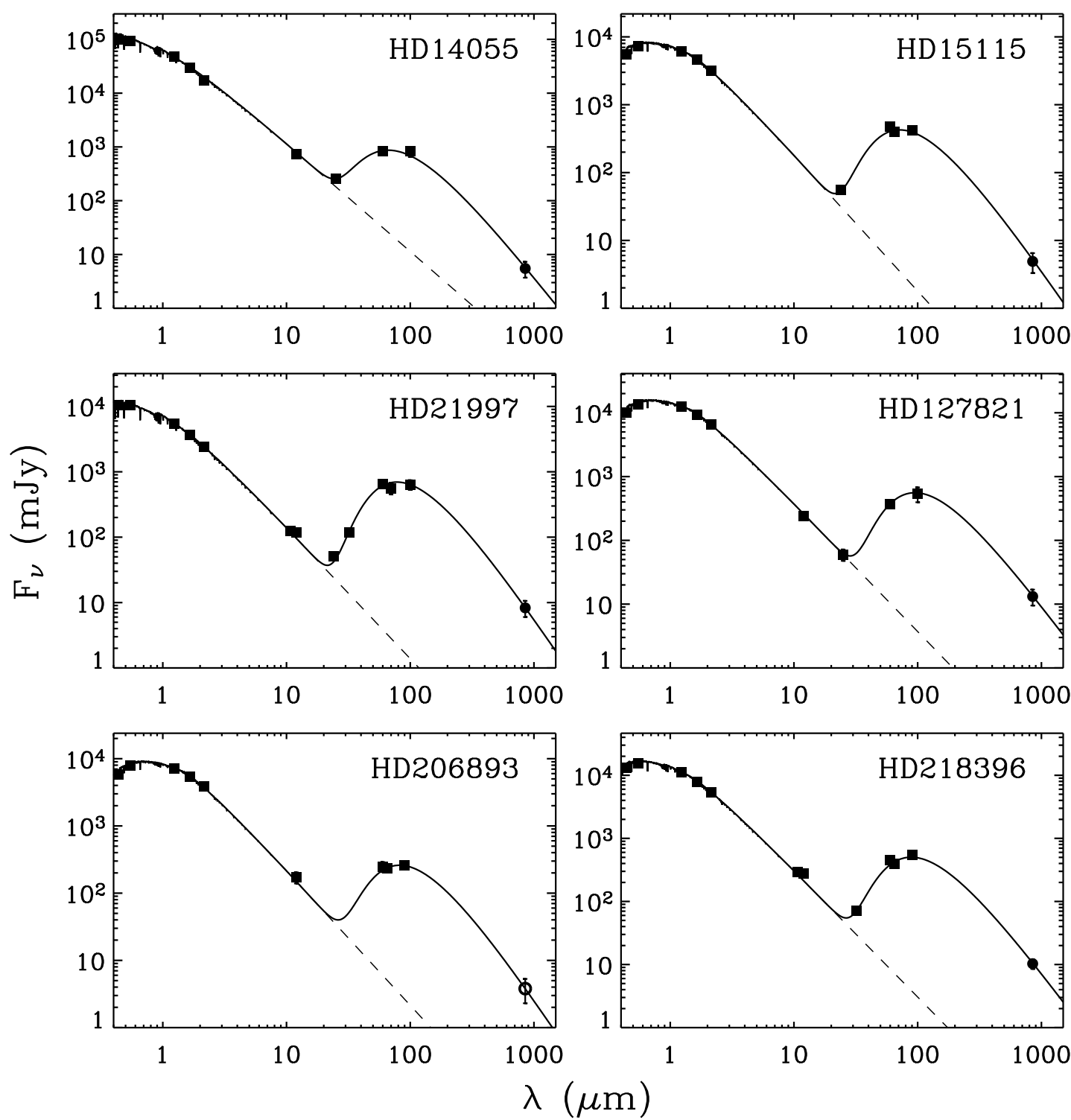

Figure 1: Spectral energy distributions for the 6 sources with SCUBA detections. The square symbols show fluxes at BVJHK bands and in the mid- and far-infrared from 12 $100 \mu \mathrm{m}$. The circular symbols show the SCUBA measurements at $850 \mu \mathrm{m}$, filled where the signal-to-noise ratio is greater than or equal to 3 , and open for the the marginal $(2.5 \sigma)$ detection of HD 206893. The solid line shows the sum of a Kurucz model of the stellar photosphere and greybody dust emission. The latter dominates at $\lambda \gtrsim 30 \mu \mathrm{m}$ and the extension of the Kurucz model is shown as a dashed line. Parameters of the greybody fits are shown in Table 2. 


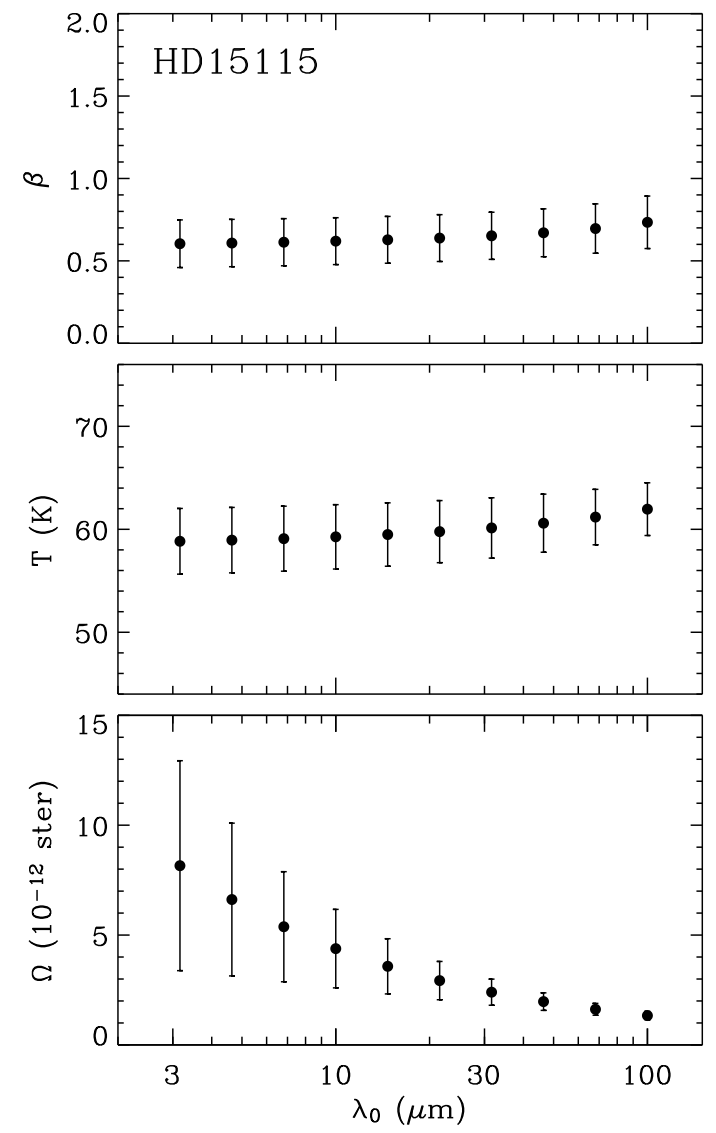

Figure 2: Variation of the best fit SED parameters with the wavelength, $\lambda_{0}$, at which the disk becomes optically thin for HD15115. The top two panels show that the dust opacity spectral index, $\beta$, and temperature, $T$, are almost independent of $\lambda_{0}$ and therefore well constrained. The lower panel shows that the dust emitting area, $\Omega$, rapidly increases as $\lambda_{0}$ decreases and is therefore poorly determined. Simliar results are found for the SED fits of the other sources. 


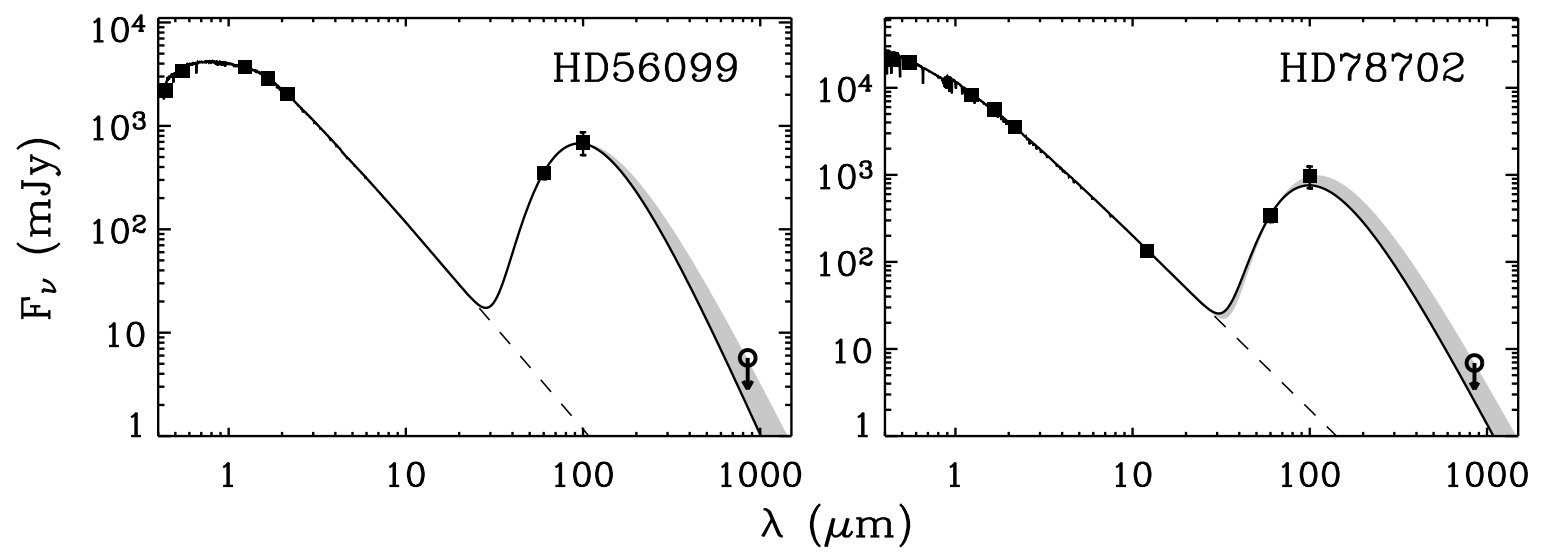

Figure 3: Spectral energy distributions for the 2 sources that were not detected by SCUBA. The data and greybody fit to the dust excess are defined as in Figure 2. The grey shaded regions show the allowable fits that are consistent with the $3 \sigma$ upper limit to the $850 \mu \mathrm{m}$ flux. Parameters of the greybody fits are shown in Table 2. 


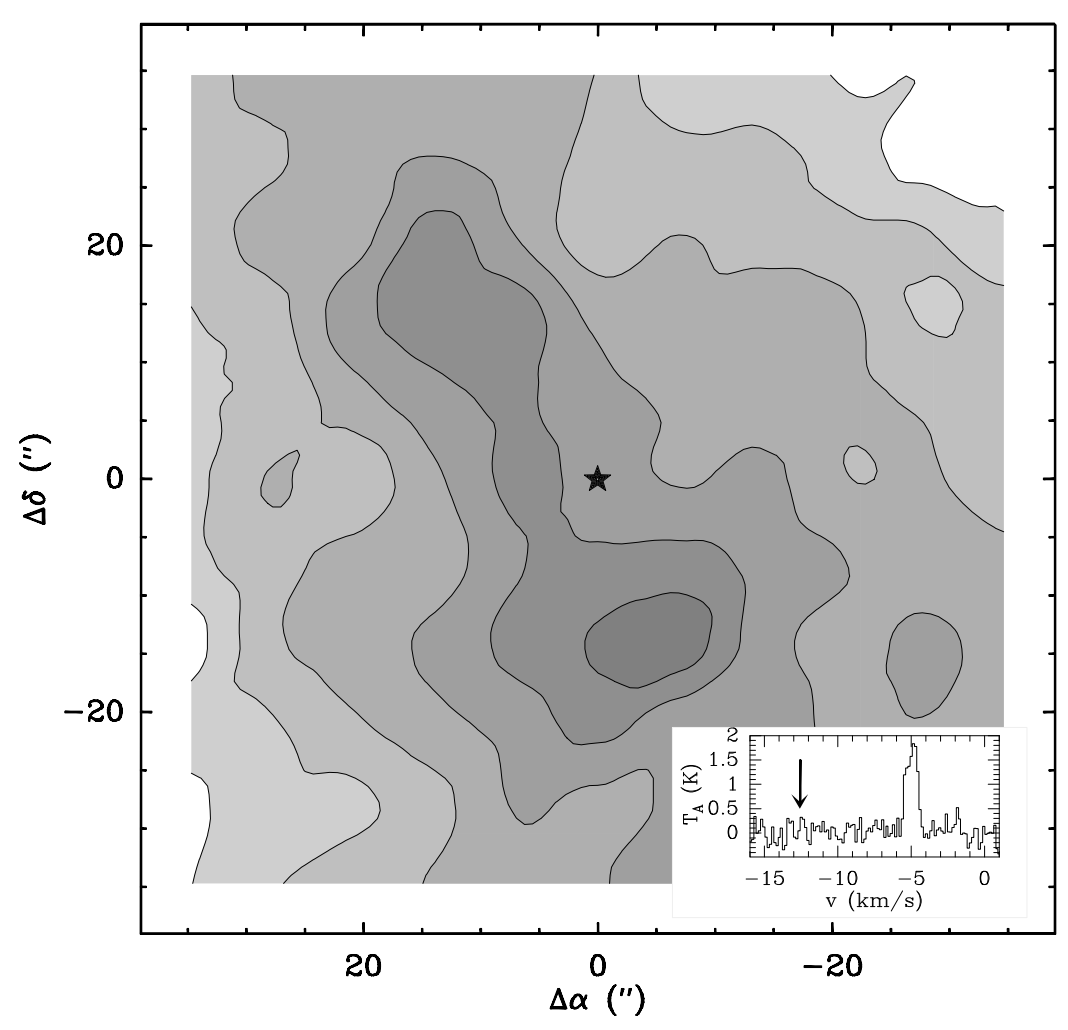

Figure 4: Contours of CO 3-2 emission toward HD 218396. The integration range is -6 to $-4 \mathrm{~km} \mathrm{~s}^{-1}$ and the contour levels are $0.25 \mathrm{~K} \mathrm{~km} \mathrm{~s}^{-1}$. The inset shows the spectrum toward the star and the arrow marks its radial velocity where any disk emission should be seen. 


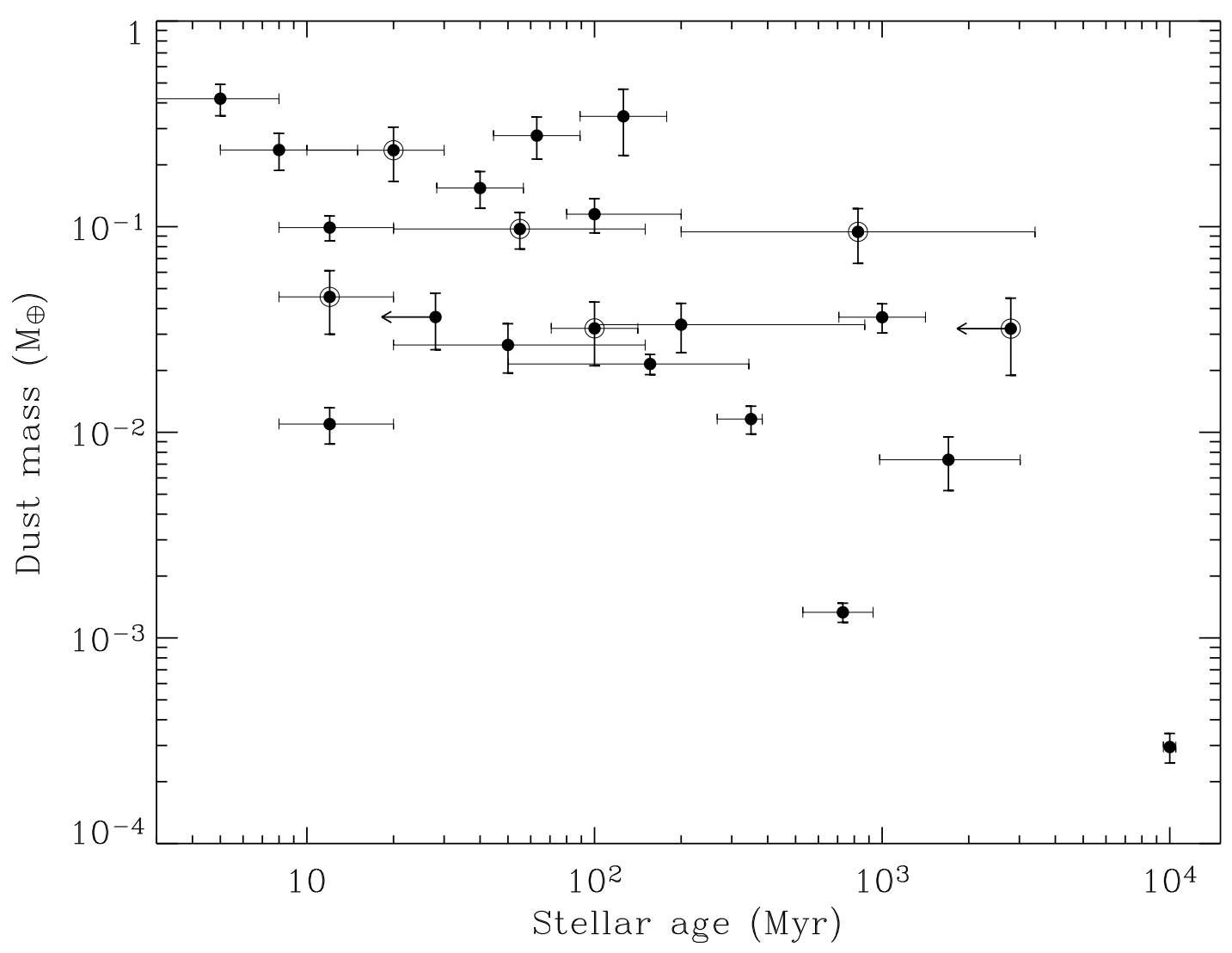

Figure 5: The dust mass in debris disks versus stellar age. These points represent the known SCUBA $850 \mu \mathrm{m}$ debris disk detections in the literature (Wyatt et al. 2003; Sheret et al. 2004; Liu et al. 2004; Greaves et al. 2004; Wyatt et al. 2005; Najita \& Williams 2005). They have been converted to a disk mass for the same mass absorption coefficient, $\kappa_{850}=1.7 \mathrm{~cm}^{2} \mathrm{~g}^{-1}$, and dust temperature given in the SCUBA detection paper. The error bars in the masses include the stated statistical uncertainty plus an assumed $10 \%$ calibration uncertainty. The error bars in the stellar ages are from Moór et al. (2006), where available, else from the discovery paper. If no error was given, a minimum and maximum age spanning a factor of two and centered on the stated age is plotted. The 6 new measurements presented in this paper are shown with an outer ring around the central dot. 